\title{
Research On Structure Modification Based Data Transmission And Security Enhancement Techniques For The Internet Of Things (Iot)
}

\author{
Wen-zhun HUANG ${ }^{1, a^{*}}$, Xin-xin XIE ${ }^{2, b}$ and Shan-wen ZHANG $^{3, c}$ \\ Department of Electronic Information Engineering, Xijing University, Xi'an 710123, China \\ ahuangwenzhun@xijing.edu.cn, ${ }^{\text {b3 } 346148500 @ q q . c o m, ~ c w j d w 716 @ 163 . c o m ~}$
}

Keywords: Internet of Things; Data Transmission; Data Security; Network Organization; Topology of the Structure; User Interface; Sensor Collection.

\section{Introduction}

Internet is an important part of the Internet in the future. The future the Internet will not only is the simple evolution of the existing Internet, in order to adapt to the future application, more need to have the new architecture, interface, data management mode and the Internet could access various heterogeneous entities and sensor etc. Future objects are identification, telecommunications, mobile, exchangeable, intelligent trusted entities can be created and destroyed and at the same time some objects can build communication network, the network and can be connected to the local and even the global other network, and can provide required services for various users. As with the Internet, mobile is as important as a network, industry experts predict that as the technology application of growing popularity, the Internet of things will be developed into the trillions of high-tech market size, for the late in the first two information wave, China runs slowly which is of great significance [1-2].

In the structure of the Internet of things and stored in the RFID tag specification which has the interoperability information, through the wireless data communication network automatically collect them to the central information system, realize the identification of the item and then through the calculation of open network to realize information exchange and sharing transparent management of goods. The Internet of things is the next big changes after the Internet. Governments, enterprises and academia have attitude towards the Internet of things to take very seriously and have a lot of countries and related organizations have long carried out the research of Internet of things and continues to this day. Ubiquitous network is based on individual and social demand, people, people and things, content and content through the on-demand access to information, information transmission, information storage, information processing which has the environment perception, perception ability and related intelligence, for individuals and society provide ubiquitous and without excluding information network services and applications. Internet of things is to point to by deploying a certain perception, calculation, executing, and communication ability of various devices, such as access to the physical world information, through the network information transmission, and processing together, so as to realize the wide-area information exchange between the people and things, and things of interconnected networks. The real application could be found in the literatures [3-7].

The current research and development has some items or physical device connected to the Internet technology, makes some researchers only from the angle of special items or physical device connected to the Internet, the one-sided understanding of the Internet of things, to a certain extent, has formed a IoT understanding erroneous zone, makes people on the Internet of things technology the understanding of the two extremes: think all network and information technology is the Internet of things technology, the Internet of things is a new opportunity in the development of information technology or think beings not connected to the Internet technology an the Internet technology is just an integration of the existing network and information technology. Due to different sensor collected information content and the representation of different time intervals and output information is different, can feel the different object and the number of sensors, lead to information fusion technology to deal with the information of multi-source heterogeneous characteristics. So, the information fusion process is to establish a unified expression and unified description of language problems, multi-dimensional information of dimension reduction optimization problem and the related problems 
of uncertain information fusion and the dispatching between the network nodes, fault-tolerant management and efficient access and so on four aspects of the problem [8].

The essence of the Internet of things must be from the virtual network world and the reality of the fusion of the physical world to explore, Internet of things is essentially a virtual world of the information network and the reality of the inevitable outcome of the physical world fusion. IoT system is virtual and real world fusion of the floorboard of the practical system. The IoT concept model based on two of the world fusion can comb some confusing concepts in the research of Internet of things and would not be enough to sum up the nature of the Internet of things, therefore, must further study IoT architecture. Dedicated Internet of things can include special IoT system and system dedicated Internet of things technology, dedicated Internet of things can be constructed for a specific applications in the field of standard technical system, also including the materials, items, information transfer, technical system and the data processing of goods and services [9]. Think in terms of extension, dedicated Internet of things the best reference to the standard of public Internet of things. But, in the primary stage of the development of the Internet of things, a dedicated Internet of things development inevitably lead to the development of the public Internet, dedicated Internet technical standards set by the not adopted by public IoT technology system, in this way, the early development of special technology of Internet of things technology might be eliminated. In the Fig.1, we show the general applications of IoT.

Dedicated Internet of things can be achieved within the scope of covering the whole country, for an application domain objects to connect to the Internet, this kind of special things can also through the Internet to provide related information services, such as international companies through the logistics network, can provide the Internet queries express the current to the location service. Between different dedicated the Internet of things, but unable to realize interconnection and interflow, unable to form the whole society needs system of Internet of things. So, we can only as information society public named IoT infrastructure, play a role similar to the Internet. The Internet of things can be divided into public Internet of things and special things which can public Internet of things refers to connect all the items, covering a social administrative area, and the public Internet connection, as a real social public information infrastructure of the Internet of things. Dedicated Internet of things refers to the connection of certain items, covering a particular area, as a special use of the Internet of things within the agency. In the Fig. 2, we show the detail.

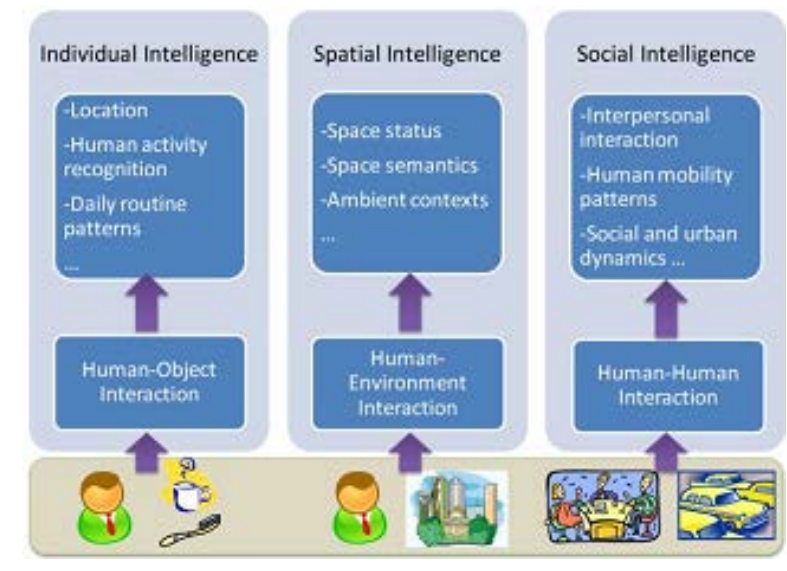

Figure 1.The General Applications of the IoT

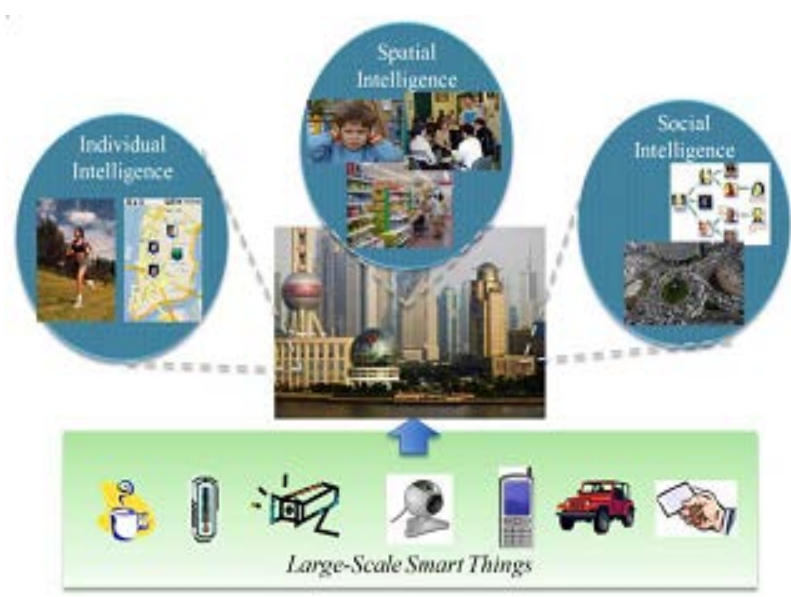

Figure 2.The Structure of the IoT Systems

In this paper, we conduct theoretical research on the structure modification based data transmission and security enhancement techniques for the Internet of Things. The Internet of things is based on microprocessor, general-purpose computer and embedded system development to the product of the advanced stage of mutual fusion. The Internet of things includes multiple disciplines, has an infinite number of application areas. In the following sections, we will discuss the issues in detail. 


\section{The The Proposed Methodology}

The IoT Structure and Organization. Both in general computer and embedded system which can be traced back to the semiconductor integrated circuit. The birth of the microprocessor for human tool provides a normalization of the kernel of intelligence. Based on microprocessor general microprocessor and embedded processors, formed the two major branches of modern computer knowledge revolution, namely the general computer and independent of the embedded system development era. General computer from wisdom platform independent development path to the Internet; Embedded system has experienced the content to the local wisdom union independent development road. Internet of things is a general-purpose computer Internet and stand-alone embedded system or the product of local content reflects the advanced stage after fusion. Today in the rapid development of Internet technology, embedded technology is particularly important [10]. At present, the embedded processor roughly divided into embedded microcontroller processor, embedded microcontroller controller and the embedded digital signal processor, embedded on a chip system. The propose Internet transmission module circuit's main function: first, will sent the data transmission to the electromagnetic wave transmitter microcontroller, then through modulation signal demodulation antenna receives baseband signal, then to micro controller.

In the process of protocol processing, Ethernet packet is first read in the cache, decide whether it is a ARP packet, if the hardware type, protocol type, and server operations; If not then judge whether the package for the IP packet, if to check the IP address is correct; If correct, then decide whether it is a ICMP packet, if call the ICMP packet protocol processing; If not then decide whether the package is a TCP packet, if call the TCP protocol, eventually combine Ethernet packets into the cache [11-13]. Support the movement of the object and the change of environment and context of the dynamic changes of the wireless link. The ability of support objects intermittent communication connection.

Wireless sensor network (WSN) is a new in the area of information science development direction. The network technology to the wireless smart sensors and the sensor is not ran a single perception of the unit. But to exchange information, he coordinated control of the organism, implementation content and content of the Internet. The perception of tentacles into every corner of the world and expanded the people access to information with the general objective world of physical information transmission network together. For people to provide the most direct, the most effective and most real information. Sensor and the perception of observer constitute the elements of a wireless sensor network. The combined with physical parameters is the forward channel sensor interface after combined with physical objects is to channel control interface; Implement a content interaction is the human-computer interaction interface which achieve the physical interaction is a communication interface. Which is decided by the material world of unlimited Internet of things has an infinite number of application areas. The Internet of things in the household life reflected in the use of information of the application of sensor devices of the organic combination of every subsystem of the household life and connected to the Internet to monitor management. People can control of home any electrical items anytime and anywhere. Compared with ordinary household, smart home not only has the function of the traditional household, in addition to provide safe and comfortable living space can also realize the household life and external information communication, make household life from passive stationary state into an intelligent perception system. RFID is a kind of using radio signals to the target object automatically and the related obtain relevant information technology. In the RFID system, the tags used to store objects of information data, tag identification information in the reading by reading and writing. In some applications, the read/write device can not only read the information stored in the tag, but also through the realization of wireless communication, speaking, reading and writing and label write data in the label.

With the development of the Internet application, the traditional label already could not meet the requirement of the increasing use of chip. In recent years, the traditional tag chip to realize the object recognition, on the basis of design a temperature sensor, switch state monitoring, power management function, and had the SPI interface of the extensible IoT tag chip has become a hot research topic. The Internet of things and cloud computing is not hype, is the integration of Internet and embedded system 
development to advanced stage. IoT covers many fields, from the perspective of the academic disciplines are needed to scientifically interpret the IoT. In the Fig. 3, we illustrate the detail.

The IoT Data Transmission and Analysis.In the Internet of things technology put forward higher request, the Internet of things technology needs further study. As we mentioned repeatedly in the thesis, the data of the Internet of things has its own characteristics, such as mass, heterogeneity, etc. In spite of all characteristics of Internet data for IoT data mining faces many difficulties, but the Internet of things is still the Internet of things technology data mining problems must be solved in the near future. The emergence of cloud computing to IoT data mining provides a good way of thinking. Currently, Hadoop is used as the basis of cloud computing by many research institutions development platform, IT can be distributed users don't understand the underlying details of distributed application development, because the Hadoop platform is open source and through the advantage of cluster provides high speed computing ability and strong storage capacity, thus can be regarded as the future like Linux system affect the IT industry. Hadoop HDFS and graphs based on distributed file system as the core, it provides low-level details transparent infrastructure system, the user can obtain good distributed computing and distributed storage programming environment.

The extension of the Internet of things is based on the Internet and the expansion of network and it will be extended and expanded to terminal between any items and items. The emergence of the computer and the Internet has created a large amount of data, the Internet of things to create the data will be far more than the Internet, the Internet contains hundreds of million level of node, on behalf of various objects, from small, ubiquitous sensor equipment, handheld devices to large network server and super computer cluster, the production of large Numbers of data every moment in, and a variety of forms, such as structured data, semi-structured data, data flow and multidimensional data, etc., have a big contribution to decision-making data, can also help small data, and the noise data, various data different nature, the means of the means of processing, storage, and on top of this information extraction method each are not identical, the data used in different systems or circumstance, reuse, or reference, such as data query, analysis, etc. Effective for such the huge amounts of data processing, storage and management is the key to the wide application of the Internet of things. XML is a commonly used technical management of semi-structured data, has since descriptive, was presented for heterogeneous data exchange, commonly used to implement data integration model lag. XML using the hierarchy of data model, through the XQuery to query the data. But it is used to describe data, such as data about data metadata is a good example, and XML is also realized by using tree structure in the operation, the important thing is can't deal with semantic information. In the Fig. 4, the data organization and structure is shown below.

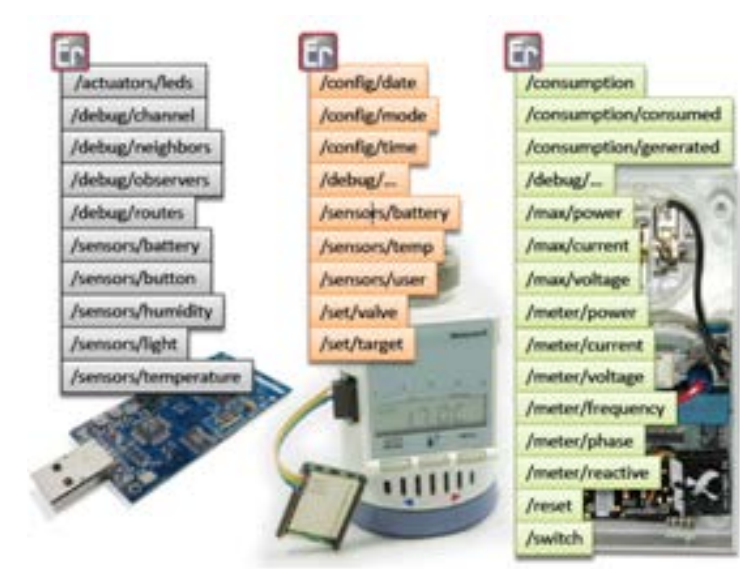

Figure 3.The Corresponding Structure of IoT

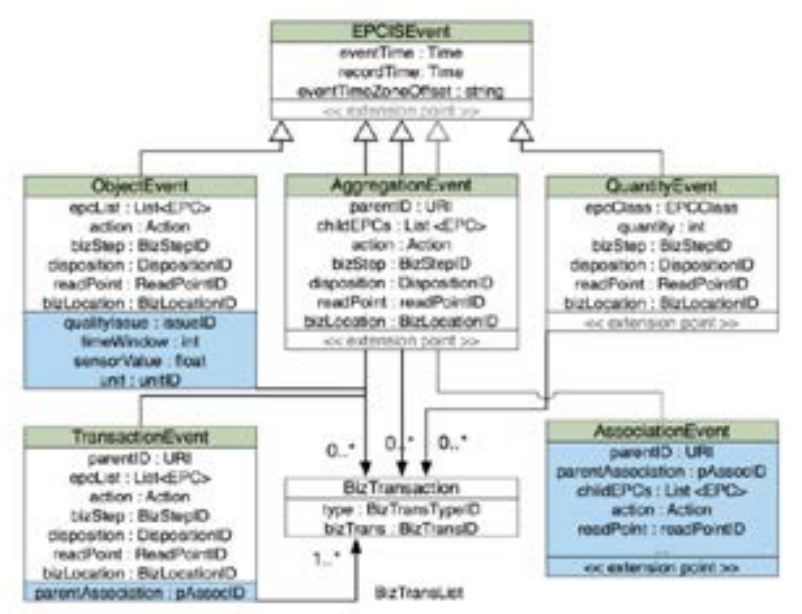

Figure 4.The Data Transmission Model for IoT 
The IoT Data Security Model.Because in the Internet of things, different objects have different data types, even the same object will use different data, so the role of data source converter is mainly to solve IoT isomerism data, it can not only ensure the integrity of the data storage, and also can guarantee the data analysis. The data security model is shown in the formula one.

$$
R O S I=\frac{A L E_{I n v}-\text { Cost }_{I n v}}{\text { Cost }}
$$

The Internet of Things (IoT) becomes an attractive research topic, in which the real entity in physical world becomes virtual entity in cyber world, and both physical and digital entities are enhanced with sensing, processing, and self- adapting capabilities to perform interaction through special addressing scheme.

The user module is the whole Internet directly using the people oriented data mining platform part, so you should have good friendly, the user can through the interface for data mining tasks, and be able to get the knowledge can be understood. In order to enhance the portability of platform, increase the open interface at the bottom of the user service module, which can make the function of the third party call IoT data mining platform, make more rich Internet application. Data mining service layer mainly includes the data preparation module, data mining engine module and user module. Data preparation module mainly includes specifications for data cleaning and transformation, data, etc. Data mining engine module mainly includes data mining algorithm, model evaluation, etc. User module mainly includes knowledge visualization of data mining. As barcode, RFID code with a string of Numbers for the manufacturer of the product and product category. The difference is that the RFID code can ensure identification and recognition of each individual item.

Intelligent identification and perception technology composed of identification and sensing technology integration and essentially has the heterogeneity, and the intelligent identification and perception of body under the different corresponding environment with different technical implementation, embodied in the related underlying implementation of different identity and sensor technology, identification technology, such as a certain environment have different implementation technologies. Similarly, sensing technology is presented in different environments. RFID tag is composed of coupling components and chips, stores the information about items need to be identified, is usually placed on the surface of the items that need to identify, it is stored information can be usually RF based read-write device through non-contact read/write access, each tag has a general global unique identification number. Therefore, the data security is more than important in the systems.

\section{Summary}

In this paper, we conduct theoretical analysis and simulation on the structure modification based data transmission and security enhancement techniques for the Internet of Things. With the development of the Internet application, the traditional label already could not meet the requirement of the increasing use of chip. We analyze the basic model and space organization of Internet of things system with detailed discussion and corresponding research. Furthermore, we combine the technique of data mining and transmission methodologies to analyze the basic pattern of data security and information hidden. The emergence of the computer and the Internet has created a large amount of data, the Internet of things to create the data will be far more than the Internet and the Internet contains hundreds of million level of node. In the future, we will conduct related numerical simulation to test the effectiveness and robustness of the proposed method.

\section{Acknowledgement}

This research was financially supported by the scientific research program of Shaanxi Provincial Education Department (Program No. 14JK2156). 


\section{References}

[1] Patton, Mark, Eric Gross, Ryan Chinn, Samantha Forbis, Leon Walker, and Hsinchun Chen, in: 2014 IEEE Joint Intelligence and Security Informatics Conference (IEEE Conference Publications, Hague 2014), p. 232.

[2] Satyanarayanan, Mahadev, Pieter Simoens, Yu Xiao, Padmanabhan Pillai, Zhuo Chen, Kiryong Ha, Wenlu Hu, and Brandon Amos: IEEE Pervasive Computing 2 Vol. 14 (2015), p. 24.

[3] Reaidy, Paul J., Angappa Gunasekaran, and Alain Spalanzani: International Journal of Production Economics Vol. 159 (2015), p. 29.

[4] Kovatsch, Matthias, Martin Lanter, and Zach Shelby, in: Proceedings of the 4th International Conference on the Internet of Things (IEEE Conference Publications, Seoul, Korea 2014), p. 1.

[5] Arias, J., Y. Barajas, and Juan Leonardo Ramirez Lopez: International Journal of Advanced Computer Science and Information Technology Vol. 3, no. 3 (2014), p.52.

[6] Wang, Haoxiang, Ferdinand Shkjezi, and Ela Hoxha, in: 2013 Sixth International Conference on Advanced Computational Intelligence (IEEE Conference Publications, Hangzhou, China 2013), p. 140.

[7] Kumar, Adarsh, Krishna Gopal, and Alok Aggarwal, in: 2014 International Conference on Parallel, Distributed and Grid Computing (IEEE Conference Publications, Taipei, Taiwan 2014), p. 423.

[8] Khodadadi, Farzad, Rodrigo N. Calheiros, and Rajkumar Buyya, in: 2015 IEEE Tenth International Conference on Intelligent Sensors, Sensor Networks and Information Processing (IEEE Conference Publications, Busan, South Korea 2015), p. 1.

[9]] Fang, Shifeng, Li Da Xu, Yunqiang Zhu, Jiaerheng Ahati, Huan Pei, Jianwu Yan, and Zhihui Liu: IEEE Trans. Industrial Informatics Vol. 10, no. 2 (2014), p. 1596.

[10] Jalali, Roozbeh, Khalil El-khatib, and Carolyn McGregor, in: 2015 18th International Conference on Intelligence in Next Generation Networks (IEEE Conference Publications, Suzhou, China 2015), p. 108.

[11] Chen, Yen-Kuang, in: 2012 17th Asia and South Pacific Design Automation Conference (Springer's LNCS Publications, Suzhou, China 2012), p. 383.

[12] Prince, Karl, Michael Barrett, and Eivor Oborn: Information and Organization Vol. 24, no. 2 (2014), p.106.

[13] Montavont, Julien, Damien Roth, and Thomas Noël: Ad Hoc Networks Vol. 14 (2014), p. 15. 